\title{
Inborn Errors of Metabolism-Clinical Findings and Laboratory Tests at the Children's Medical Center, Tehran, Iran
}

\author{
Farzaneh Abbasi, ${ }^{1,2,3}$ Aria Setoodeh, ${ }^{1,2,3,}$ Fatemeh Sayarifard, ${ }^{1,2,3}$ Parastoo Rostami, ${ }^{1,2,3}$ and Ali \\ Rabbani $\mathbf{i}^{1,2,3}$ \\ ${ }^{1}$ Growth and Development Research Center, Tehran University of Medical Sciences, Tehran, IR Iran \\ ${ }^{2}$ Children's Medical Center, Pediatrics Center of Excellence, Tehran, IR Iran \\ ${ }^{3}$ Department of Pediatrics, Tehran University of Medical Sciences, Tehran, IR Iran \\ "Corresponding author: Aria Setoodeh, Children Medical Center, Pediatrics Center of Excellence, Tehran, IR Iran. E-mail: arset59@yahoo.com
}

Received 2016 February 07; Revised 2016 June 18; Accepted 2016 July 22.

\begin{abstract}
Background: Inborn errors of metabolism (IEM) are disorders caused by genetic mutations of the normal metabolic pathways. Objectives: The aim of this study was to investigate clinical findings and laboratory tests for IEMs to determine a proper clinical and laboratorial approach to these disorders.

Methods: This descriptive study involved 180 patients with suspected IEM at the children's medical center (CHM) in Tehran. The patients' information was recorded in special forms, and three levels of examinations were performed. Data were subjected to frequency, percent, and chi square tests using SPSS version 17.

Results: The study sample included 87 (48.3\%) female patients and 93 (51.7\%) male patients. The most common (20\%) clinical presentation of patients with IEMs was poor feeding or failure to thrive, and the frequency of the condition in children younger than age 1 year was approximately $35 \%$. Eighty percent of patients received a definitive diagnosis by the first and second level of examination. The most common (17\%) disorders were proponic acidemia, methylmalonic acidemia (14\%), and urea cycle disorder (14\%).

Conclusions: Considering the non-specific primary presentation of IEMs and the importance of clinical diagnosis other than specific laboratory tests, it is necessary to develop a general guide for an approach to IEM that provides appropriate management for high-risk families and infants.
\end{abstract}

Keywords: Inborn Errors of Metabolism, Dysmorphy, Genetic Mutations, Iran

\section{Background}

Inborn errors of metabolism (IEM) are disorders caused by genetic mutations which block the normal metabolism of complex molecules. The number of IEMs in humans known to be attributed to inherited enzyme defects in normal metabolic pathways exceeds 500 (1). IEMs cause different signs and symptoms, such as like metabolic acidosis, nausea, failure to thrive, and developmental delays, and physical manifestations, such as cardiomegaly, skeletal dysplasia, and encephalopathy (2, 3 ). The age of the manifestation of symptoms varies from early childhood to adulthood (4). Although each disorder is rare, the aggregate rate of IEM is significant, at 1 of every 500 births (5). Among children with mental disabilities, the rate of IEM is approximately 5.75\% (6).

The wide spectrum of clinical manifestation of IEMs and the complexity of the diagnosis challenges clinicians (7). In many cases, prevention of mortality and neurologic sequelae depends on early diagnosis and proper treatment at an early age (8). Recent advances in laboratory methods, pre-birth screening, and early diagnosis and treat- ment have significantly reduced the morbidity and mortality rates (9-11). The most common disorders are Wilson's disease, glycogen storage disease, mucopolysaccharidosis, and galactosemia (12).

IEMs are single-gene disorders as a mutation in one gene blocks some points along the metabolic pathways. Inheritance of these defects is often autosomal recessive (13), although other types of inheritance have been documented (e.g., autosomal dominant, X-related) (14). However, the prevalence of IEM is higher in countries where the practice of cousin marriage is common, and children inherit 1.4 to 1.8 of parents' genes (15). In Iran, the rate of cousin marriage is higher than in other countries, so the incidence of IEMs might be higher but has not been estimated yet. At the same time, there is no united approach to IEM, and patients with inappropriate diagnosis are treated imperfectly. The statistics for inherited metabolic disorders and the long-term potential for them in Iran indicate the need for more accurate studies on the incidence, common manifestations, appropriate approach, and ways to reduce IEMs. 


\section{Objectives}

The aim of this study was to investigate clinical findings and laboratory tests concerning IEMs to determine a proper clinical and laboratorial approach to the disorders.

\section{Methods}

This descriptive study involved patients with suspected IEMs who had not received a definitive diagnosis and were referred to the children's medical center (CHM) in Tehran from 2011 to 2013. The cumulative prevalence of IEMs in individuals younger than age 20 years is approximately 40 in 100,000 births (7). The estimated sample size according to the formula was about 60 patients. To gain this sample size, the number of suspected patients in the study should be three times larger, or 180.

The inclusion criteria were cousin marriage; family history of IEM; history of sudden unknown death in the family; history of psychological disorders; seizures; unreasonable decreases in blood sugar; encephalopathy; protein intolerance; self-harm behaviors; sepsis without the risk factor of an infection and severity of symptoms inappropriate for the type of infection; dysmorphy and developmental delays; ocular defects, such as cataracts and pigmented retinopathy; secondary respiratory distress due to unknown metabolic acidosis; respiratory alkalosis accompanied by neural disorders; hypertrophic cardiomyopathy; repeated vomiting; nausea; malnutrition; failure to thrive; hypertrophy of the liver and spleen; cirrhosis; liver failure; obstructive jaundice; kidney stones in children; urine with an unusual odor; chronic muscular weakness and pain; ichthyosis; photosensitivity; attacks of lethargy; changes in consciousness; mental disabilities; irritability; coma; developmental delays, especially a lack of neurologic or psychologic development in adolescents or children older than 5 years; structural anomalies in brain; hypotonia and hypertonia; apnea; and laboratory tests for hypoglycemia; hyperammonemia; acidosis; increased level of amino acids and organic acids; and reduced substance levels in urine.

The exclusion criteria were parents' refusal, diagnosis of non-metabolic disease in primary tests, and lack of follow-up by parents. The incidence of inherited metabolic disorders differs by the age at which symptoms (16), so quota sampling was performed in children younger than 15 years old. Demographic information and laboratory test results were recorded in special forms.

After selection of patients according to these criteria and obtaining consent to participate in the study, three levels of examination were performed (17). First-level examinations consisted of clinical examination, genetic coun- seling to check the family history, drawing the family tree, and estimating the first level of laboratory tests (Table 1). Patients diagnosed with any disorder other than IEM were excluded from the study.

Table 1. Laboratory Examinations in Patients With Suspected IEMs

\begin{tabular}{|c|c|}
\hline Level & Laboratory Examinations \\
\hline \multirow{10}{*}{ First level } & Complete blood count \\
\hline & Electrolytes and serum uric acid \\
\hline & Anion gap \\
\hline & Arterial blood gases \\
\hline & Blood glucose \\
\hline & Plasma ammonia and arterial blood \\
\hline & Liver function tests \\
\hline & Urine reducing substances \\
\hline & Urine ketones \\
\hline & CSF lactate \\
\hline \multirow{7}{*}{ Second level } & Urinary organic acids \\
\hline & Urinary amino acids \\
\hline & Plasma uric acid \\
\hline & Plasma amino acids \\
\hline & Acyl carnitine profile \\
\hline & Biotinidase assay \\
\hline & CSF amino acid analysis \\
\hline \multirow{2}{*}{ Third level } & Enzyme assay in blood cells or skin fibroblas \\
\hline & DNA mutation assay \\
\hline
\end{tabular}

The second level of examinations consisted of more accurate, complementary laboratory tests used to diagnose the exact type of IEM. Finally, diagnoses were verified by the third level of laboratory tests, which consisted of enzymatic and genetic assessment.

Data were analyzed using SPSS version 17. Chi-square and Fisher exact tests were used to assess qualitative variables.

All the procedures were performed in accordance with the ethical standards of the responsible institutional and national committees on human experimentation and with the Helsinki Declaration of 1975 , as revised in 2000 . Informed consent for inclusion in the study was obtained from all patients.

\section{Results}

The present study involved 180 patients with suspected IEMs, including 87 (48.3\%) female and 93 (51.7\%) male patients. Sixty (33.3\%) patients received definite diagnoses of IEMs. In all, 102 (61.2 \%) patients were younger than 1 year old, and the rest were older. Table 2 shows the descriptive characteristics of the patients. The most common clinical manifestations in patients with a verified diagnosis of IEM were poor feeding or failure to thrive (20\%) and lethargy or 
coma (16.7\%). Figure 1 shows the frequency of clinical signs and symptoms of IEMs in this study. Definitive diagnoses were made in 43 (80\%) patients by general tests (first and second level) and in the rest of patients (20\%) by more specialized tests.

\begin{tabular}{|c|c|}
\hline Characteristics & No. (\%) \\
\hline \multicolumn{2}{|l|}{ Gender } \\
\hline Female & $87(48.3)$ \\
\hline Male & $93(51.7)$ \\
\hline \multicolumn{2}{|l|}{ Age } \\
\hline$<1$ & $109(61.2)$ \\
\hline $1-5$ & $51(28.7)$ \\
\hline $5-10$ & $13(7.3)$ \\
\hline $10-15$ & $4(2.2)$ \\
\hline$>15$ & $1(0.6)$ \\
\hline \multicolumn{2}{|l|}{ Laboratory Tests } \\
\hline First level & $63(37.3)$ \\
\hline Second level & $88(52.1)$ \\
\hline Third level & $18(10.7)$ \\
\hline
\end{tabular}

The clinical manifestations of disorders and the test results for male and female patients of different ages were not significantly different. However, in the patients younger than 1 year old, who were the largest group (50\%), one-third (34.2\%) of primary clinical presentations were poor feeding or failure to thrive. Seizures (14\%) and hypotonia or hypertonia (12\%) were the second and third most common clinical presentations. In patients older than 1 year, the most common clinical presentations were skeletal malformations and dysmorphic features.

Episodic decompensation, poor feeding or failure to thrive, splenomegaly and hepatosplenomegaly occurred more frequently in female patients than male ones. Skeletal malformations, dysmorphic features, lethargy or coma, and abnormal hair and/or dermatitis occurred more frequently in male patients (Figure 2). In this study, the most prevalent IEMs were propionic acidemia (17\%), methylmalonic acidemia (14\%), and urea cycle disorder (14\%). Figure 3 shows the frequency of the different types of IEM in this study.

\section{Discussion}

In the present study, the most common clinical presentation of patients with IEMs was poor feeding or failure to thrive, which had a frequency of $35 \%$ in patients younger than 1 year old. In $80 \%$ of patients, diagnoses were established by first- and second-level tests. In this study, the most common disorders were propionic acidemia, methylmalonic acidemia, and urea cycle disorder. Other studies have also reported propionic acidemia and methylmalonic acidemia as the most prevalent disorders (18).

During infancy, most IEMs are accompanied by nonspecific symptoms that are not effective in diagnosis, and an accurate diagnosis can only be obtained by conducting more specialized laboratory tests. In general, IEMs are categorized in three groups according to clinical presentation. The first group includes cases with disturbances in the catabolism or synthesis of complex molecules. The resulting disorders are progressive, permanent, and not related to alimentary ingestion.

The second group consists of errors in the intermediary metabolism that result in acute and recurrent intoxication from the aggregation of blocked toxic metabolites. The common symptoms and signs are dehydration, metabolic acidosis, vomiting, and lethargy. The clinical expression is intermittent, and the disorders worsen over time and are not related to alimentary ingestion.

The third group is energy deficiency diseases that result from defects in the metabolism of food and the production or utilization of energy from food. This group of disorders has systemic symptoms and signs due to disturbances in metabolic pathways, such as energy deficiencies and the accumulation of toxic metabolites.

IEMs, such as popionic acidemia, methylmalonic acidemia, and urea cycle disorder, the most common disorders in this study, belong to the second group that can cause acute, life-threatening symptoms in early infancy. The disorders are accompanied by protein intolerance, so they are worsened by breastfeeding. Consequently, the most important symptoms, especially in younger infants, are poor feeding and lethargy. This result is confirmed by other studies $(19,20)$.

Choudhuri et al found that the predominant symptoms and signs of IEMs are poor feeding, failure to thrive, and feeding intolerance (21). However, in the study by Jailkhani et al, the most common clinical presentations were seizures (30\%), acidosis, and hypoglycemia (15\%) (22).

In this study, $80 \%$ of patients were diagnosed by the first and second group of tests. What is important in the request for a special test for an IEM diagnosis is the correct clinical approach and decision by the clinician based on the symptoms, family history, examination, common laboratory tests, and knowledge of IEMs and their differences (23). A definitive diagnosis requires specialized enzyme assays and the identification of molecular defects, which are not widely available in clinics. These tests are used when there is high clinical suspicion of a specific kind of disor- 


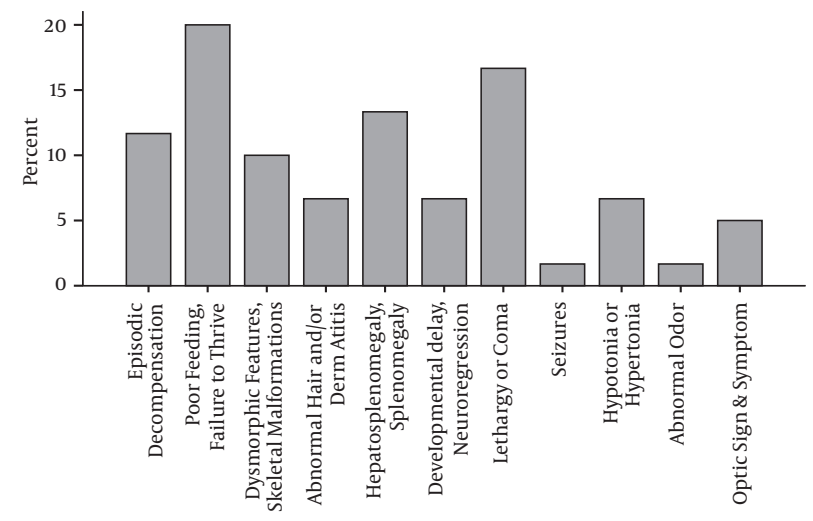

Figure 1. Clinical findings for IEMs

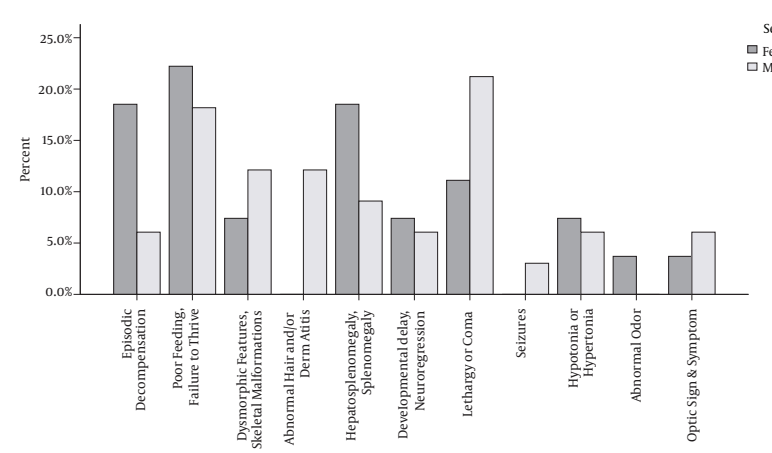

Figure 2. IEM Clinical Findings by Gender

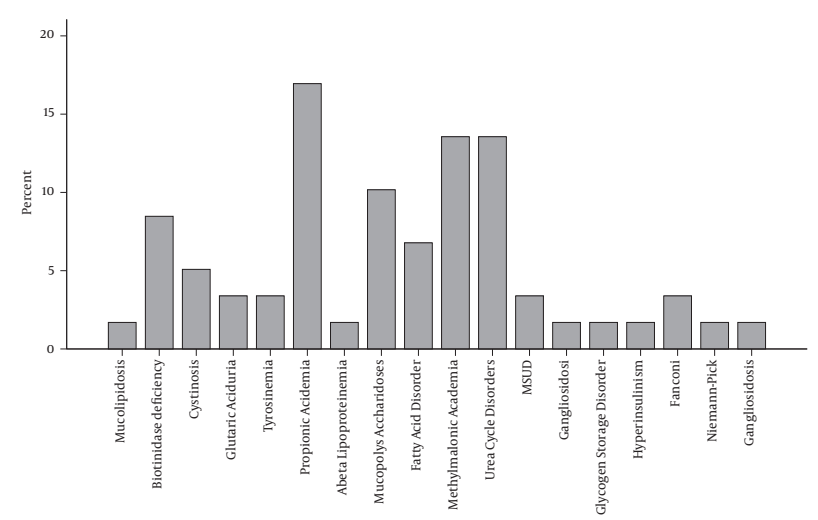

Figure 3. Disease type of IEMs

$\operatorname{der}(24)$.

Therefore, considering the non-specific primary presentations and the importance of clinical diagnosis other than specific laboratory tests, it is necessary to develop a general guide to approach IEMs that provides appropriate management for high-risk families and infants.
IEMs represent a wide spectrum of disorders, and their precise investigation requires more diverse patients. A larger sample is recommended for future studies. 


\section{Footnotes}

Authors' Contribution: Farzaneh Abbasi was the principal investigator, conceived of the idea for the study, and wrote the draft of manuscript. Aria Setoodeh was also a principal investigator, participated in the study design and coordination, and performed the supervision. Ali Rabbani also helped conceived of the idea of the study, draft the manuscript, and perform the statistical analysis. Fatemeh Sayarifard and Parastoo Rostami collected the data.

Funding/Support: This study was supported by a grant from the growth and development research center, Tehran University of Medical Sciences.

\section{References}

1. Childs B, Valle D, Jimenez-Sanchez G. In: The metabolic and molecular basis of inherited disease. 8 ed. Scriver CR, Beaudet AL, Sly WS, Valle D, editors. McGraw-Hill; 2001. The Inborn error and biochemical variability.

2. Garrod AE. Inborn errors of metabolism. Oxford; 1909.

3. Nia S. Psychiatric signs and symptoms in treatable inborn errors of metabolism. J Neurol. 2014;261 Suppl 2:S559-68. doi: 10.1007/s00415014-7396-6. [PubMed: 25145892].

4. Cleary MA, Green A. Developmental delay: when to suspect and how to investigate for an inborn error of metabolism. Arch Dis Child. 2005;90(11):1128-32. doi: 10.1136/adc.2005.072025. [PubMed: 16243864].

5. Oliveira A, Santos A, Martins A, D'Almeida V. Screening for inborn errors of metabolism among newborns with metabolic disturbance and/or neurological manifestations without determined cause. Sao Paulo Med J. 2001;119(5):160-4.

6. ICMR collaborating centres and central co-ordinating unit. Multicentric study on genetic causes of mental retardation in India. Indian J Med Res. 1991;94:161-9. [PubMed: 1879891].

7. Low LCK. Inborn errors of metabolism: clinical approach and management. Hong Kong Med J. 1996;2(3):274-81.

8. Garrod AE, Harris H. Inborn errors of metabolism. Oxford University Press; 1963. p. 170.

9. Al Riyami S, Al Maney M, Joshi SN, Bayoumi R. Detection of inborn errors of metabolism using Tandem mass spectrometry among high-risk Omani patients. Oman Med J. 2012;27(6):482-5. doi: 10.5001/omj.2012.115. [PubMed: 23226820].

10. Huang X, Yang L, Tong F, Yang R, Zhao Z. Screening for inborn errors of metabolism in high-risk children: a 3-year pilot study in Zhejiang Province, China. BMC Pediatr. 2012;12:18. doi: 10.1186/1471-2431-1218. [PubMed: 22364411].

11. Ozben T. Expanded newborn screening and confirmatory follow-up testing for inborn errors of metabolism detected by tandem mass spectrometry. Clin Chem Lab Med. 2013;51(1):157-76. doi: 10.1515/cclm2012-0472. [PubMed: 23183752].

12. Verma IC. Burden of genetic disorders in India. Indian J Pediatr. 2000;67(12):893-8. [PubMed: 11262988].

13. Milunsky A, Littlefield JW. The prenatal diagnosis of inborn errors of metabolism. Annu Rev Med. 1972;23:57-76. doi: 10.1146/annurev.me.23.020172.000421. [PubMed: 4264784].

14. Hamamy H, Alwan A. Genetic disorders and congenital abnormalities: strategies for reducing the burden in the region. East Mediterr Health J. 1997;3(1):123-32.

15. Bittles $\mathrm{AH}$. The role and significance of consanguinity as a demographic variable. Popul Dev Rev. 1994:561-84.

16. Moammar H, Cheriyan G, Mathew R, Al-Sannaa N. Incidence and patterns of inborn errors of metabolism in the Eastern Province of Saudi Arabia, 1983-2008. Ann Saudi Med. 2010;30(4):271-7. doi:10.4103/02564947.65254. [PubMed: 20622343].

17. Sharma S, Kumar P, Agarwal R, Kabra M, Deorari AK, Paul VK. Approach to inborn errors of metabolism presenting in the neonate. Indian JPediatr. 2008;75(3):271-6. [PubMed: 18376097].

18. Burton BK. Inborn errors of metabolism: the clinical diagnosis in early infancy. Pediatrics. 1987;79(3):359-69. [PubMed: 3547297].

19. Burton BK. Inborn errors of metabolism in infancy: a guide to diagnosis. Pediatrics. 1998;102(6):69. [PubMed: 9832597].

20. Raghuveer TS, Garg U, Graf WD. Inborn errors of metabolism in infancy and early childhood: an update. Am Fam Physician. 2006;73(11):1981-90. [PubMed: 16770930].

21. Choudhuri T, Sengupta S. Inborn error of metabolism-an Indian perspective. Int J Hum Genetics. 2006;6(1):89.

22. Jailkhani R, Patil VS, Laxman HB, Shivashankar AR, Kulkarni SP, Ravindra MS. Selective screening for inborn errors of metabolism in children: single centre experience from Karnataka. J Clin Diagn Res. 2008;2(4):952-8.

23. Waber L. Inborn errors of metabolism. Pediatr Ann. 1990;19(2):105-912-3, 17-8. [PubMed: 2407993].

24. Seashore MR, Rinaldo P. Metabolic disease of the neonate and young infant. Semin Perinatol. 1993;17(5):318-29. [PubMed: 8290974]. 